\begin{tabular}{c|c|c}
\hline ISSN 2525-4812 (versão online) & Revista Terceira \\
$\begin{array}{c}\text { ISSN 2238-7641 (versão impressa) } \\
\text { http://www.revistaterceiramargem.com/ } \\
\text { index.php/terceiramargem/index }\end{array}$ & $\begin{array}{c}\text { Recebido em: 19/6/2019 } \\
\text { Aprovado em: } 30 / 2 / 2020\end{array}$ & $\begin{array}{c}\text { Revisaço } \\
\text { Margem Amazônia }\end{array}$ \\
\hline
\end{tabular}

Como citar o artigo:

PINTO, L. M. R. B; FRAXE, T. J, P; SILVA, M. L. Um breve panorama da bioprospecção: sua origem, suas definições, potencial econômico e status-quo no Brasil. Revista Terceira Margem Amazônia, v. 6, n.15, p. 90-102, 2020. Doi: http://dx.doi.org/10.36882/2525-4812.2020v6i15p90-102.

\title{
UM BREVE PANORAMA DA BIOPROSPECÇÃO: SUA ORIGEM, SUAS DEFINIÇÕES, POTENCIAL ECONÔMICO E STATUS-QUO NO BRASIL
}

\author{
Leonardo Marcelo dos Reis Braule Pinto ${ }^{I}$
}

\author{
Therezinha de Jesus Pinto Fraxe ${ }^{2}$ \\ Michele Lins Aracaty e Silva ${ }^{3}$
}

\begin{abstract}
Resumo: A Sustentabilidade é um dos temas mais debatidos no mundo na era do século XXI, e, no caso do Brasil, tudo fica muito mais frágil, visto que nos primeiros dias da nova gestão, os representantes do Governo Federal alteraram as atribuições formais dos ministérios, produzindo a mais drástica reestruturação dos órgãos de primeiro escalão desde o governo Collor (1990-1992). A conclusão é de que as políticas socioambientais brasileiras, construídas em 40 anos de avanços, foram colocadas em xeque. Assim, podese dizer que a Bioprospecção é uma das ferramentas de rentabilidade e sustentabilidade mais eficiente que existe e dada a importância do assunto, ela será explicada neste trabalho bem como se demonstrará seu estado atual no Brasil.
\end{abstract}

Palavras-Chave: Bioprospecção; Sustentabilidade; Brasil; Economia.

Abstract: The Sustainability is one of the most debated topics in the world in the 21 st century era, and in the case of Brazil, everything is much more fragile, since in the early days of the new administration, representatives of the Federal Government changed the formal attributions of ministries, producing the most drastic restructuring of the senior bodies since the Collor administration (1990-1992). The conclusion is that Brazilian social and environmental policies, built on 40 years of progress, were put in check. Thus, it can be said that Bioprospecting is one of the most efficient profitability and sustainability tools that exists and given the importance of the subject, it will be explained in this paper as well as its current state in Brazil.

Keywords: Bioprospecting; Sustainability; Brazil; Economy.

\footnotetext{
1 Mestrando no Programa de Pós-Graduação em Ciência e Tecnologia para Recursos Amazônicos (PPGCTRA/UFAM)

2 Professora Titular da Faculdade de Ciências Agrárias da Universidade Federal do Amazonas (FCA/UFAM)

${ }^{3}$ Docente do Departamento de Economia e Análise da Universidade Federal do Amazonas (UFAM)
} 


\title{
INTRODUÇÃO
}

A palavra Bioprospecção vem da junção do radical grego - bio, que significa vida, com a palavra que vem do latim prospectione, que significa olhar à frente, muito usada como ferramenta de planejamento estratégico de futuro, principalmente no mercado financeiro onde se precisa pensar estrategicamente para se obter os melhores ganhos, os maiores lucros, as melhores vantagens.

A partir disso, se pode dizer que a palavra Bioprospecção significa planejar estrategicamente se utilizando dos recursos biológicos, ou seja, significa em outras palavras, gerar riqueza a partir de recursos naturais. O termo Bioprospecção, foi formalmente cunhado apenas em 1993 como sendo "a exploração da biodiversidade para a descoberta de recursos genéticos e substâncias bioquímicas comercialmente úteis (LAIRD, 2002)". Para Saccaro Junior (2011A, p. 7) a Bioprospecção pode ser,

\begin{abstract}
(...) definida como a busca sistemática por organismos, genes, enzimas, compostos, processos e partes provenientes de seres vivos em geral, que possam ter um potencial econômico e, eventualmente, levar ao desenvolvimento de um produto. É relevante para uma ampla gama de setores e atividades, incluindo biotecnologia, agricultura, nutrição, indústria farmacêutica e de cosméticos, biorremediação, biomonitoramento, saúde, produção de combustível por meio de biomassa, entre outros. Os alvos da Bioprospecção são coletivamente chamados de recursos genéticos. Seu conjunto forma o patrimônio genético nacional.
\end{abstract}

Dito isto, vale ressaltar a importância sustentável que a Bioprospecção possui em essência, pois além de contribuir com a geração de riqueza, contribui também com a preservação ecológica, visto que se utiliza dos recursos ambientais e não do patrimônio ecológico ${ }^{1}$, e ainda estimula o desenvolvimento social já que necessita de tecnologia e pessoal capacitado para desenvolvimento de produtos.

Vale ressaltar que entre a infinidade de finalidades da Bioprospecção, o desenvolvimento de novos fármacos tem-se mostrado com importante destaque, devido à sua relação com a saúde e bem-estar humanos, ao mesmo tempo em que se destaca também com as cifras econômicas envolvidas no processo mercadológico. O mercado farmacêutico mundial movimentou em 2008 mais de US\$ 700 bilhões e em 2014 esse

\footnotetext{
${ }^{1}$ Segundo a UNESCO, patrimônio ecológico ou natural é a fonte do recurso natural, ou seja, é donde o ser humano retira o recurso para atender as suas necessidades. É geralmente não renovável ou demora muito mais tempo para se renovar que o recurso. Ex: fruto é recurso natural, enquanto a árvore que o fornece é patrimônio. Disponível em: https://es.unesco.org/themes/patrimonio-natural, Acesso em 08 de agosto de 2019.
} 
valor atingiu cerca de US\$1,1 trilhão, sendo a maior parte do crescimento na demanda proveniente de países emergentes (IMS, 2010, apud. SACCARO JUNIOR, 2011A). Valese dizer que o papel da Bioprospecção no setor é fundamental: cerca de $50 \%$ dos fármacos atuais foram desenvolvidos com base em moléculas biológicas. No caso de drogas anticâncer e antibióticos, essa proporção pode ser ainda maior, chegando próximo aos 70\% (UNU-IAS,2005, apud. SACCARO JUNIOR, 2011A). Esses números se refletem nos mercados: mais da metade das 150 drogas mais prescritas nos Estados Unidos durante a década 2000-2010 foram derivativos de recursos naturais, sem degradação de patrimônio ecológico (GRIFO et al., 1997, apud. SACCARO JUNIOR, 2011A).

\section{REFERENCIAL TEÓRICO}

Dado o grande potencial econômico que o patrimônio ecológico possui, os países em desenvolvimento, que por sua vez, concentram a maior parte da biodiversidade mundial, começaram, nas últimas décadas do século passado, a discutir a ideia de propriedade sobre os recursos genéticos e os conhecimentos tradicionais associados aos mesmos. O Brasil foi um dos atores mais ativos nas negociações multilaterais decorrentes. Foi considerada injusta a situação em que o acesso aos recursos genéticos era livre em qualquer lugar, mas os produtos obtidos daí eram objetos de apropriação monopolística, principalmente por meio de patentes, por empresas sediadas na maioria dos casos em países desenvolvidos, que possuem maior aparato tecnológico, financeiro e informacional. Uma nova visão se consolida com a Convenção sobre Diversidade Biológica (CDB), que reconhece a soberania de cada país sobre os recursos genéticos localizados em seu território.

Ao mesmo tempo, são reconhecidos os direitos das comunidades tradicionais e indígenas sobre seu saber. Tal saber deve ser acessado apenas com o consentimento das comunidades envolvidas, e com elas, deve haver uma repartição justa dos benefícios auferidos, principalmente os econômicos (CBD, [s.d.], apud. SACCARO JUNIOR, 2012).

No caso do Brasil, o maior entrave ao desenvolvimento da atividade bioprospectiva está intimamente relacionado ao ambiente regulatório. E este, para Saccaro Junior (2011A), deriva de características socioculturais internas associadas a um contexto mundial de mudanças recentes na forma de se encarar o patrimônio natural. 
Pode se dizer que, a bioprospecção é uma atividade inserida na cultura brasileira. Registros arqueológicos indicam que os indígenas utilizavam plantas de tabaco, outras medicinais, e ainda várias para a construção de moradias e de canoas antes da chegada dos colonizadores. O consumo intensivo de moluscos por indígenas na costa do país levou à formação de sambaquis, literalmente "monte de conchas", os quais eram utilizados na fabricação de objetos como raspadores e facas de pedra, batedores e suportes do mesmo material (DEAN, 2010, apud. BERLINCK, 2012).

Os índios também caçavam aves para utilizar suas penas como ornamentos e como moeda de troca com outros produtos de necessidade pessoal, principalmente alimento. Com a chegada dos europeus, as atividades de bioprospecção se intensificaram. A primeira foi a exploração da madeira nobre de várias espécies de árvores, sendo o Pau-Brasil a mais conhecida. Estima-se que nos primeiros anos de exploração do Pau-Brasil foram derrubadas cerca de 1.200 toneladas/ano de madeira, o que corresponderia a cerca de 2 milhões de árvores entre 1500 e 1600 (DEAN, 2010, apud. BERLINCK, 2012).

Entre 1859 e 1861 foi instituída a Comissão Científica do Império, a primeira formada exclusivamente por pesquisadores genuinamente brasileiros. O projeto dessa comissão foi conhecer e relatar diversos aspectos da natureza e cultura do sertão do Ceará, e, em menor extensão, Pernambuco e Paraíba, dentre os quais extensa variedade da flora e fauna locais, bem como "conhecimentos tradicionais e populares (...) sobre virtudes medicinais de plantas e animais". A publicação comentada dos documentos dessa comissão inclui riquíssima iconografia que apenas recentemente foi divulgada em bela edição extensamente ilustrada (KURY, 2009, apud. BERLINCK, 2012).

No fim do século XIX, três dos primeiros institutos de pesquisa criados em território brasileiro desenvolviam atividades ligadas, direta ou indiretamente, à atividade Bioprospectiva: a Imperial Estação Agronômica de Campinas (1887), o Instituto Soroterápico Federal - atual Fiocruz - (1900) e o Instituto Butantan (1901). A vocação da pesquisa brasileira para atividades que envolvem Bioprospecção se firmou com a criação do Instituto de Química Agrícola no Jardim Botânico do Estado do Rio de Janeiro. Desta etapa participaram diretamente aqueles que seriam os fundadores dos estudos em química de produtos naturais no Brasil: Affonso Seabra, Paulo Lacaz, Walter Mors, Benjamin Gilbert e Otto Richard Gottlieb. Em paralelo, investigações desenvolvidas na Faculdade de Medicina de Ribeirão Preto (SP) por Maurício Oscar da Rocha e Silva e Sergio Ferreira levaram à descoberta de peptídeos bioativos da jararaca, que seriam mais 
PINTO, L. M. R. B; FRAXE, T. J, P; SILVA, M. L.

tarde utilizados como matriz para desenvolver um dos principais fármacos para $\mathrm{o}$ tratamento da hipertensão: o captopril. (BERLINCK, 2012).

Este breve histórico omite muitas outras áreas de desenvolvimento da ciência brasileira, diretamente ligadas à Bioprospecção, tais como a bioquímica, a biologia molecular, a microbiologia e a biotecnologia, por exemplo, mas dá um breve panorama do potencial e importância da atividade bioprospectiva (BERLINCK, 2012).

Os mais diversos trabalhos de pesquisadores nos primeiros 60 anos do século XX levou à formação de uma verdadeira escola de Bioprospecção brasileira, da qual nos dias de hoje participam dezenas, quiçá centenas, de cientistas ambientais em todo o país, distribuídos por todas as regiões, presentes nas principais Universidades e institutos de pesquisa do país, buscando-se a conhecer, entender e explorar racionalmente os recursos naturais como plantas, animais e microrganismos para a produção de substâncias e desenvolvimento de processos com as mais diversas atividades e utilidades, principalmente na busca de desenvolver produtos com viabilidade produtiva e potencial econômico para atender a demanda nacional e internacional (BERLINCK, 2012).

Uma decorrência óbvia dessa intensa atividade de Bioprospecção foi o firmamento de várias indústrias de cosméticos e fármacos que buscam aproveitar elementos da biodiversidade brasileira para a produção de cosméticos, medicamentos, aditivos alimentares, cremes de pele, e outros produtos de imensa importância econômica, ambiental e biológica. O Acheflan, desenvolvido e produzido pelo laboratório Aché, foi o primeiro exemplo de fitofármaco brasileiro a entrar no seleto mercado farmacêutico com sucesso em suas expectativas produtivas e de demanda (BERLINCK, 2012).

A afirmativa sobre propriedade incidida aos recursos genéticos e conhecimentos tradicionais associados é relativamente recente, tendo começado a ser debatida apenas nas últimas décadas do século passado. Até então o acesso era indiscriminado e sem ressalvas, podendo ser realizado por todos, em qualquer lugar do globo. Essa situação se transforma com a Convenção sobre Diversidade Biológica (CDB), que reconhece a soberania de cada país sobre os recursos genéticos localizados em seus limites geográficos nacionais (CDB, 2010). A mudança é decorrente da procura de países em desenvolvimento - vale destaque para o Brasil nas negociações multilaterais -, que concentram a maior parte da biodiversidade mundial, por terem considerado injusta a situação em que o livre acesso aos recursos genéticos era permitido, mas os produtos obtidos daí eram objetos de apropriação monopolística, principalmente por meio de 
patentes, por empresas sediadas na maioria dos casos em países já desenvolvidos, principalmente da Europa, Ásia e América do Norte (SACCARO JUNIOR, 2011B).

A CDB é um acordo internacional, lançado durante a Conferência das Nações Unidas sobre o Meio Ambiente e Desenvolvimento, em 1992, em paralelo a outros documentos multilaterais, como a Convenção sobre o Clima e a Agenda 21, que juntos devem nortear a relação entre os países, o ambiente e o desenvolvimento econômico. Tem como ideia central a conservação e a utilização sustentável, justa e equitativa da biodiversidade, reconhecendo a importância desses objetivos para o desenvolvimento e a sobrevivência da humanidade, bem como a responsabilidade social do ser Humano sobre a contemporânea trajetória de diminuição da diversidade biológica do globo. Estabeleceu também as diretrizes que devem ser seguidas para conciliar conservação e desenvolvimento. Assinada e ratificada pela maioria dos países pertencentes à Organização das Nações Unidas (ONU), foi promulgada no Brasil pelo Decreto de número 519/1998. Desde sua entrada em vigor, o patrimônio genético tornou-se uma riqueza nacional, cabendo a cada país legislar sobre as formas de acesso e de repartição dos benefícios gerados pelo uso das mesmas (SACCARO JUNIOR, 2011B).

Para Saccaro Junior (2011B, p. 231),

\begin{abstract}
O conhecimento do ambiente acumulado ao longo do tempo por comunidades indígenas e tradicionais, denominado conhecimento tradicional, também foi levado em consideração pela CDB: ele tem uma íntima relação com o processo de Bioprospecção, servindo como guia. As informações que essas comunidades fornecem sobre as propriedades de plantas e outros organismos são valiosas, muitas vezes imprescindíveis, para a seleção de alvos de pesquisa. Até então, enquanto o conhecimento das empresas farmacêuticas era protegido pela propriedade intelectual, o conhecimento tradicional era entendido como algo público, de livre acesso. Por esse motivo, a CDB reconhece também os direitos das comunidades tradicionais e indígenas sobre seu saber: este deve ser acessado apenas com o consentimento das comunidades envolvidas, e com elas deve haver uma repartição justa dos benefícios gerados.
\end{abstract}

Vale ressaltar que a partir da CDB, alguns países começaram a legislar sobre o assunto e o Brasil, país que detém o maior patrimônio genético do mundo, está atrás da Costa Rica na capacidade de gerar renda com o uso da biodiversidade e seus recursos. É visível que um arcabouço legal inadequado pode levar os bioprospectores a pesquisar em outros países, em que as condições sejam mais palpáveis e de fácil atendimento, ou ainda a praticar biopirataria, visto que praticamente inexistem mecanismos internacionais efetivos de fiscalização e sanção aos biopiratas. Na verdade, a incerteza associada à possibilidade de tais mecanismos serem implementados por si só constitui fonte de dilemas à regulamentação, porque a adequação desta pode mudar em função de variáveis 
externas. Neste cenário, cabe a países como a China, a Índia e o Brasil, portadores de grande porcentagem da biodiversidade mundial, com papel de liderança nas negociações da Conferência das Partes (COP-órgão de decisão e implementação da CDB, composto por todos os governos e organizações de integração econômica regional que a tenham ratificado), aprimorarem sua regulamentação interna para que ela se torne um balizador das decisões e normas internacionais a respeito do assunto (SACCARO JUNIOR, 2011A).

Buscando viabilizar o cumprimento do que foi estabelecido na CDB o governo brasileiro criou o Programa Nacional de Biodiversidade - PRONABIO, no ano de 1994, que tem como objetivo estimular a parceria entre o Estado e a sociedade civil no uso sustentável dos recursos naturais. O PRONABIO veio a estabelecer duas formas de financiamento, são elas: o Projeto de Conservação e Utilização Sustentável da Diversidade Biológica Brasileira- PROBIO e o Fundo Brasileiro para a Biodiversidade FUNBIO. O PROBIO consiste numa espécie de financiamento do governo e tem como objetivo incentivar a criação de projetos que estabeleçam parcerias entre os setores privado e público da nossa sociedade, promovendo informações sobre a biodiversidade brasileira e seu uso de forma equilibrada. O FUNBIO, por sua vez, é o maior fundo privado de biodiversidade já estabelecido à nível nacional (CUNHA, SANTOS, BRAGA JUNIOR, 2008).

Vários documentos internacionais foram autenticados pelo Brasil com a finalidade de proteger a diversidade biológica, como exemplo tem-se a Convenção para Proteção da Flora, da Fauna e das Belezas Naturais dos Países da América, em 1940; a Convenção Internacional para Conservação do Atum do Atlântico, em 1966; a Convenção Relativa às Zonas Úmidas de Importância Internacional, Particularmente como "Habitats" de Aves Aquáticas, em 1971; a Convenção Para o Comércio Internacional das Espécies da Flora e Fauna Selvagens em Perigo de Extinção, em 1973 (ANTUNES, 2010).

Segundo Saccaro Junior (2011A, p. 11),

As primeiras iniciativas brasileiras para regulamentar a matéria ocorreram em 1995, com o PL no 306/1995, substituído em 1998 pelo PL no 4.842/1998, quando também outros dois PLs (nos 4.579/1998 e 4.751/1998) foram apresentados sobre o assunto. Estes e outros projetos relacionados ainda tramitavam na Câmara dos Deputados em 2000, quando um contrato entre a Organização Social Bioamazônia e a empresa farmacêutica Novartis foi duramente criticado pela imprensa. Devido a tais críticas, em sua maioria relacionada à inexistência de legislação que protegesse adequadamente os recursos genéticos existentes em território nacional, o contrato acabou por não ser executado. Porém, a repercussão negativa do fato levou à edição da Medida Provisória (MP) no 2.052, de 29 de junho de 2000, em vigor atualmente sob o no 2.186-16/2001. Esta determina que o acesso ao conhecimento tradicional 
associado e ao patrimônio genético existente no país, bem como sua remessa para o exterior, somente sejam efetivados mediante autorização da União, e institui como autoridade competente para esse fim, o Conselho de Gestão do Patrimônio Genético (CGEN) (AZEVEDO, 2005). A MP também regulamenta a repartição dos benefícios derivados do uso do patrimônio genético, bem como acesso à tecnologia e à transferência de tecnologia para sua conservação e utilização, sendo atualmente o principal marco regulatório para a Bioprospecção.

Abaixo, segue quadro que resume o panorama da Bioprospecção e seu desenvolvimento técnico, científico e Jurídico no Brasil.

Quadro 1 - Panorama Histórico da Bioprospecção no Brasil

\begin{tabular}{|c|c|c|c|c|}
\hline SÉCULO & $(\ldots)-\mathrm{XVI}$ & XVI-XVII & XVIII-XIX & XX-XXI \\
\hline ACONTECIMENTO & $\begin{array}{c}\text { Uso de } \\
\text { sambaquis } \\
\text { pelos } \\
\text { indígenas. }\end{array}$ & $\begin{array}{l}\text { Exploração, por } \\
\text { parte dos } \\
\text { colonos } \\
\text { europeus, da } \\
\text { Seiva de plantas, } \\
\text { principalmente o } \\
\text { Pau Brasil. }\end{array}$ & $\begin{array}{c}\text { Criação de } \\
\text { Institutos e } \\
\text { Comissões } \\
\text { que } \\
\text { pesquisem } \\
\text { sobre meio } \\
\text { ambiente e } \\
\text { seu uso } \\
\text { econômico. }\end{array}$ & $\begin{array}{c}\text { Realização de } \\
\text { pesquisas } \\
\text { bioprospectivas } \\
\text { em } \\
\text { Universidades e } \\
\text { Institutos de } \\
\text { Todo País. } \\
\text { Realização da } \\
\text { CDB e criação } \\
\text { de Órgãos e } \\
\text { legislação } \\
\text { nacional sobre o } \\
\text { tema. }\end{array}$ \\
\hline
\end{tabular}

Fonte: Elaboração própria.

A interligação entre o acesso ao recurso natural, a repartição de benefícios com os detentores do conhecimento tradicional e a propriedade intelectual do pesquisador é outro ponto crucial ainda não resolvido de forma clara no País. A MP n 2.186- 16/2001 obrigava, para o requerimento de patentes relacionadas a produtos oriundos da biodiversidade, que seja informada a origem do material genético pesquisado e do conhecimento tradicional associado à ele, quando for a situação, a fim de garantir que o acesso se dê na forma do previsto em lei. Essa obrigatoriedade foi regulamentada somente em 2006, pelo Instituto Nacional de Propriedade Industrial (Inpi), por meio da Resolução Inpi $n^{\circ} 134 / 2006$ (ainda vigente). Esta obriga a declaração, por parte do requerente de patente, no momento de seu depósito, da data e do número da autorização concedida pelo 
CGEN (Conselho de Gestão do Patrimônio Genético), para acesso ocorrido após a publicação da MP (INPI, 2009, apud. SACCARO JUNIOR, 2011A).

O que é um problema é que uma vez que entre esta obrigação e a resolução passaram-se mais de cinco anos, muitos pedidos ocorridos neste período se tornaram irregulares. Ainda mais, a demora no trâmite e na concessão da autorização pelo CGEN passou a ser mais prejudicial ao inventor, pois adiar o pedido da patente pode significar prejuízo, principalmente financeiro. Na prática, muitos interessados que estavam com solicitações pendentes no CGEN entraram com pedido de patente no Inpi, mesmo que sem o número, por medo de perderem a prioridade sobre a invenção. Para solucionar tal questão, o instituto publicou as Resoluções ns 207 e 208/2009 (ainda vigentes), desobrigando o solicitante de informar, no ato do depósito, a data e o número da autorização dada pelo CGEN. Esta informação poderá ser enviada ao Inpi até o exame da patente, quando poderá ocorrer uma exigência para regularizar a situação. Mas novas instruções não resolvem a situação de pedidos que acessaram a biodiversidade antes da publicação da Medida Provisória. Nestes casos, permaneceram em situação irregular e o Inpi trabalha em conjunto com o CGEN para discutir uma legislação que resolva definitivamente essa questão (INPI, 2009, apud. SACCARO JUNIOR, 2011A).

Vale ressaltar que, também, o CGen, durante a $3^{\text {a }}$ Reunião Extraordinária, realizada no dia 31 de outubro de 2018, aprovou a Resolução CGen n ${ }^{\circ} 19$, de 2018, para estabelecer forma alternativa para a regularização dos usuários que realizaram atividades de acesso ao patrimônio genético ou ao conhecimento tradicional associado, unicamente para fins de pesquisa científica (Art. 38, $\S 2^{\circ}$ da Lei $n^{\circ} 13.123$ ). Mas que também não beneficiou quem deu entrada nos processos antes da Vigência da Resolução.

Entretanto, falando do panorama acadêmico, pode-se dizer que o Brasil conta atualmente com uma vantagem significativa: já possui um setor acadêmico bem desenvolvido. Prova disso é o sucesso obtido pelas redes integradas de pesquisa, na realização dos projetos sobre o genoma brasileiro, em que vários laboratórios e centenas de pesquisadores se uniram para a produção e interpretação de dados genéticos de várias espécies (NEGRAES; EGLER, 2002). A pós-graduação strictu-sensu do país passa por intenso processo de crescimento, diversificação e amadurecimento, tendo atingido uma escala e um padrão de qualidade que a distingue entre as nações em desenvolvimento e a coloca no páreo com as Universidades de países ricos. Isso é comprovado pelo crescimento de cerca de $1.000 \%$ ocorrido no número de doutores titulados anualmente entre 1987 e 2008, chegando a mais de 10 mil anuais, o que representa um quinto do total 
de doutores formados nos EUA. As áreas de ciências biológicas, agrárias e da saúde, mais intimamente relacionadas à Bioprospecção, foram responsáveis por mais de $40 \%$ dos doutores formados entre 1996 e 2008 (CGEE, 2010).

\begin{abstract}
A melhor forma de conseguir manejar sustentavelmente a diversidade biológica é estimulando a educação ambiental e, consequentemente, a construção de uma consciência coletiva conservacionista. Para isso é necessário que haja uma efetiva integração entre o Poder Público, as ONG's e as instituições públicas e particulares voltadas à pesquisa científica, as comunidades tradicionais, enfim, todos que possuem legítimo interesse (CUNHA, SANTOS, BRAGA JUNIOR, 2008, p. 04).
\end{abstract}

Em outras palavras, a educação é a forma mais eficiente de se gerar consciência e atitudes responsáveis que sejam eficientes na busca pelo desenvolvimento sustentável, e nesse quesito, o Brasil anda à passos rápidos, o que é bastante positivo para o mesmo no panorama de busca em conciliar desenvolvimento e sustentabilidade.

Nos dias atuais, no Brasil, a MP n² 2186-16/2001 foi revogada e deu lugar a Lei de ${ }^{\circ} 13.123$, de 20 de maio de 2015, a qual dispõe sobre o acesso ao patrimônio genético, sobre a proteção e o acesso ao conhecimento tradicional associado e sobre a repartição de benefícios para conservação e uso sustentável. A Lei nº 13.123, de 2015, entrou em vigor em 16 de novembro de 2015, sendo regulamentada pelo Decreto $\mathrm{n}^{\circ} 8.772$, de 11 de maio de 2016.

A Lei $n^{0} 13.123 / 2015$ teve sua gênese no Projeto de Lei $n^{0} 7.735 / 2014$, que tramitou no Congresso Nacional, sendo muito discutida entre os órgãos do Governo vinculados à área ambiental e bioprospectiva, a Empresa Brasileira de Pesquisa Agropecuária - EMBRAPA, o Grupo de Coalizão formado pelas Indústrias Farmacêuticas e Cosméticas, a Confederação Nacional da Indústria - CNI, a Confederação da Agricultura e Pecuária do Brasil - CNA e a Federação das Indústrias do Estado de São Paulo - FIESP. Durante as discussões em torno do Projeto de Lei e também durante a votação na Câmara dos Deputados, verificou-se a pouca participação das Universidades, das comunidades indígenas, comunidades tradicionais e camponeses. Estes três últimos grupos, divulgaram carta de repúdio, ratificada por 54 organizações, onde condenam o PL e a sua forma de tramitação (CIMI, 2014, apud. MENUCHI et. al., 2016).

O que ocorreu por conta da fragilidade com que a legislação trata a repartição dos recursos oriundos do uso do conhecimento tradicional. Outro fator negativo ainda é o 
processo burocrático que o pesquisador necessita enfrentar para fazer uso dos recursos bioprospectivo o que dificulta e desencoraja esse pesquisador (MENUCHI et. al., 2016).

\section{RESULTADOS}

Fica claro que o país ainda necessita trabalhar a questão da regulamentação da atividade bioprospectiva de forma a não prejudicar sua essência sustentável ao mesmo tempo em que à torna uma atividade economicamente viável e competitiva a nível Nacional e Internacional, o que, na verdade é um grande desafio não apenas para o Brasil, mas para o mundo.

\section{REFERÊNCIAS}

ANTUNES, P. B. Direito ambiental. 12. ed. Rio de Janeiro: Lumen Juris, 2010.

BERLINCK, Roberto Gomes de Souza. Bioprospecção no Brasil: um breve histórico. Cienc. Cult. vol.64 no.3 São Paulo 2012. Disponível em: http://cienciaecultura.bvs.br/pdf/cic/v64n3/a10v64n3.pdf, Acessado em 09 de agosto de 2019.

CBD - CONVENTION ON BIOLOGICAL DIVERSITY. History of the convention. [s.d.]. In: SACCARO JUNIOR, Nilo. Como Impulsionar a Bioprospecção no Brasil: bases para uma moderna regulação do acesso a recursos genéticos e ao conhecimento tradicional associado. Rio de janeiro: IPEA, 2012. Disponível em:http://www.ipea.gov.br/portal/images/stories/PDFs/TDs/td_1807.pdf, Acessado em 05 de Agosto de 2019.

CBD - CONVENTION ON BIOLOGICAL DIVERSITY (2010). Convention on Biological Diversity. Disponível em: https://www.cbd.int/2011-2020/2010. Acessado em: 15 jun. 2019.

CENTRO DE GESTÃO E ESTUdOS ESTRATÉGICOS (CGEE). Doutores 2010: estudos da demografia da base técnico-científica brasileira. Brasília, 2010. Disponível em: http://www.cgee.org.br. Acesso em: 15 jun. 2019.

CIMI. Conselho Indígena Missionário. Assessoria de Comunicação. Comunidades manifestam repúdio à $\mathrm{PL}$ sobre uso de conhecimentos tradicionais e recursos genéticos. Conselho Indigenista Missionário, Brasília, 11 nov. 2014.In: MENUCHI, Luciana Nalim Silva; et. al. O novo marco legal para acesso ao patrimônio genético e proteção do conhecimento tradicional associado. Revista GEINTEC - ISSN: 2237-0722. São Cristóvão/SE - 2016. Vol. 6/n. 1/ p.2954-2965. Disponível em: http://revistageintec.net/index.php/revista/article/view/802/645, Acessado em 12 de Agosto de 2019. 
CUNHA, Francisco Assis Bezerra da; SANTOS, Esdras Brito dos; BRAGA JUNIOR, Francisco Assis Teixeira. Bioprospecção: marcos legais para a proteção da biodiversidade e normatização do acesso ao patrimônio genético e seus derivados. Disponível em: http://www.urca.br/ered2008/CDAnais/pdf/SD3_files/Francisco_CUNHA.pdf, Acessado em 12 de agosto de 2019.

DEAN, W. A ferro e fogo - a história e a devastação da Mata Atlântica brasileira. Companhia das Letras, São Paulo, 2010. In: BERLINCK, Roberto Gomes de Souza. Bioprospecção no Brasil: um breve histórico. Cienc. Cult. vol.64 no.3 São Paulo 2012. Disponível em: http://cienciaecultura.bvs.br/pdf/cic/v64n3/a10v64n3.pdf, Acessado em 09 de agosto de 2019.

GRIFO, F. et al. The origins of prescription drugs. In: GRIFO, F.; ROSENTHAL J. (Org.). Biodiversity and Human Health. Washington, DC: Island Press, 1997. p. 131-163. In: SACCARO JUNIOR, Nilo. Desafios da Bioprospecção no Brasil. Rio de janeiro: IPEA, 2011. Disponível em: http://www.ipea.gov.br/portal/index.php?option=com_content\&view=article \&id=7066, Acessado em 05 de junho de 2019.

INSTITUTO NACIONAL DA PROPRIEDADE INDUSTRIAL (INPI). Inpi altera regras para facilitar pedidos de patentes ligados à biodiversidade. Rio de Janeiro, 2009. Acesso em: 15 jul. 2010. In: SACCARO JUNIOR, Nilo. Desafios da Bioprospecção no Brasil. Rio de janeiro: IPEA, 2011A. Disponível em: http://www.ipea.gov.br/portal/index.php?option=com_content\&view=article \&id=7066, Acessado em 05 de junho de 2019.

INTERCONTINENTAL MARKETING SERVICES (IMS). Health. Acesso em: 15 jun. 2010. In: SACCARO JUNIOR, Nilo. Desafios da Bioprospecção no Brasil. Rio de janeiro: $\quad$ IPEA, 2011. Disponível em: http://www.ipea.gov.br/portal/index.php?option=com_content\&view=article\&id=7066, Acessado em 05 de junho de 2019.

KURY, L. (Org.), Comissão científica do Império. Andrea Jakobsson Estúdio Editorial Ltda, Rio de Janeiro, 2009. In: BERLINCK, Roberto Gomes de Souza. Bioprospecção no Brasil: um breve histórico. Cienc. Cult. vol.64 no.3 São Paulo 2012. Disponível em: http://cienciaecultura.bvs.br/pdf/cic/v64n3/a10v64n3.pdf, Acessado em 09 de agosto de 2019.

LAIRD, S. A. in Biodiversity and traditional knowledge - equitable partnerships in practice, Edited by Sarah A. Laird, Earthscan Publications Ltd., London \& Sterling (USA), p. 22, 2002.

MENUCHI, Luciana Nalim Silva; et. al. O novo marco legal para acesso ao patrimônio genético e proteção do conhecimento tradicional associado. Revista GEINTEC - ISSN: 2237-0722. São Cristóvão/SE - 2016. Vol. 6/n. 1/ p.2954-2965. Disponível em: 
http://revistageintec.net/index.php/revista/article/view/802/645, Acessado em 12 de Agosto de 2019.

NEGRAES, C. L. B.; EGLER, P. C. G. The Brazilian Genome Project: a successful example of a research network. Science Technology \& Society, v. 7, n. 2, 2002.

UNITED NATIONS UNIVERSITY. INSTITUTE OF ADVANCED STUDIES (UNUIAS). Report: Bioprospecting in Antartica, 2005. Acesso em: 15 jun. 2010. In: SACCARO JUNIOR, Nilo. Desafios da Bioprospecção no Brasil. Rio de janeiro: IPEA, 2011. Disponível em: http://www.ipea.gov.br/portal/index.php?option=com_content $\&$ view=article \&id=7066, Acessado em 05 de junho de 2019.

SACCARO JUNIOR, Nilo. Como Impulsionar a Bioprospecção no Brasil: bases para uma moderna regulação do acesso a recursos genéticos e ao conhecimento tradicional associado. Rio de janeiro: IPEA, 2012. Disponível em :http://www.ipea.gov.br/portal/images/stories/PDFs/TDs/td_1807.pdf, Acessado em 05 de Agosto de 2019.

SACCARO JUNIOR, Nilo. Desafios da Bioprospecção no Brasil. Rio de janeiro: IPEA, 2011A. Disponível em: http://www.ipea.gov.br/portal/index.php?option=com_content \&view=article \&id=7066, Acessado em 05 de junho de 2019.

SACCARO JUNIOR, Nilo. A regulamentação de acesso a recursos genéticos e repartição de benefícios: disputas dentro e fora do Brasil. Ambiente \& Sociedade. Campinas v. XIV, n. 1. p. 229-244. jan.-jun. 2011B. Disponível em: http://www.scielo.br/pdf/asoc/v14n1/a13v14n1.pdf. Acessado em 10 de julho de 2019. 